\title{
AC Supply Design of Metal Particle Detection Sensor
}

\author{
Wenjun Zhao ${ }^{1}$, Haiying Jiang ${ }^{1, \text { a }}$, Zhigang $\mathrm{Si}^{1}$ \\ ${ }^{1}$ School of Information Science and Engineering, University of Jinan, City Jinan, China \\ ${ }^{a}$ ise_jianghy@ujn.edu.cn
}

KEY WORDS: AC power supply, microcontroller, SPWM, frequency conversion

Abstract. This design introduces an adjustable frequency AC Supply which is used as the excitation source of the metal particle detection sensor. According to different sine table, STC15W4K16S4 microcontroller outputs corresponding frequency SPWM control signals. These signals are amplified by the MOSFET drivers. Then they drive the H-bridge inverter circuit. After the RC low-pass filter, the output frequency is from $2400 \mathrm{~Hz}$ to $3000 \mathrm{~Hz}$, and the frequency interval is $50 \mathrm{~Hz}$. The output wave is sine AC voltage. This system can be used as the excitation source of metal wear particle detection sensor.

\section{Introduction}

This design is applied to a subject study of metal wear particle detection, as an excitation source of reverse double excitation metal particle detection sensor. According to literature [1] and preliminary simulation experiment [2], the output of the sensor is greatly influenced by the excitation frequency. When the excitation frequency range is from $2000 \mathrm{~Hz}$ to $3000 \mathrm{~Hz}$, its output signal is stronger, and the output signal intensity increases with the excitation voltage.

\section{Circuit Design of AC Supply System}

H-bridge inverter circuit design. The MOSFET which is comprised of the H-bridge selected by Infineon produced IRF540N type MOSFET. The circuit is shown in Figure 1.

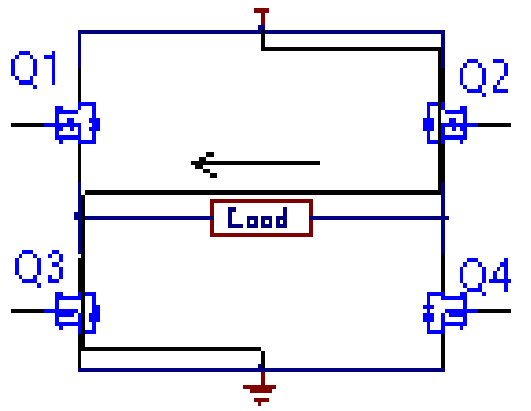

(a)

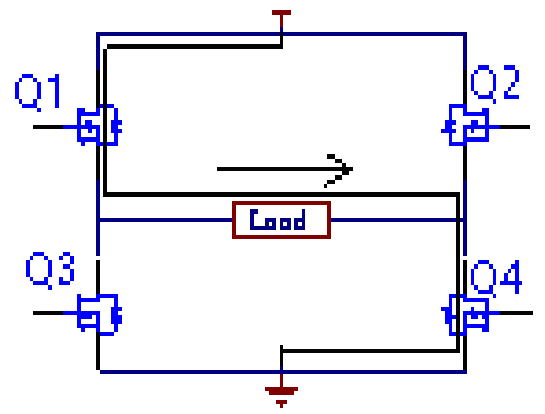

(b)

Fig.1 H-bridge inverter circuit

As shown in Figure1 (a), when the PWM control signal of the MOSFET Q2 and Q3 is high level, the control signal of Q1 and Q4 is low level. The Q2 and Q3 on the diagonal of the H-bridge arm are turned on and the Q1 and Q4 on the other side of the H-bridge arm are turned off. The current flows from the DC power supply, Q2, load, Q3 to ground. The current direction of the load is 
indicated by the arrows in the figure, from right to left. Assuming the current direction is positive at this time. Similarly shown in Figure1 (b), when the PWM control signal of Q2 and Q3 is low level, the control signal of Q1 and Q4 is high level. The current direction is negative at this time. Thus, by controlling the two diagonal MOSFETs turn on or off to achieve the load current alternately positive and negative, that is, to achieve the DC to AC conversion.

MOSFET drive circuit design. As MOSFET IRF540N in the H-bridge need more than 10 volts of the gate-source voltage to saturation conduction, the signal sent by the microcontroller cannot directly drive the MOSFETs, it must be amplified to 10 volts or more by the driver. The drivers use IR2101 which is a high-voltage, high-power, dual-channel MOSFET driver [3].

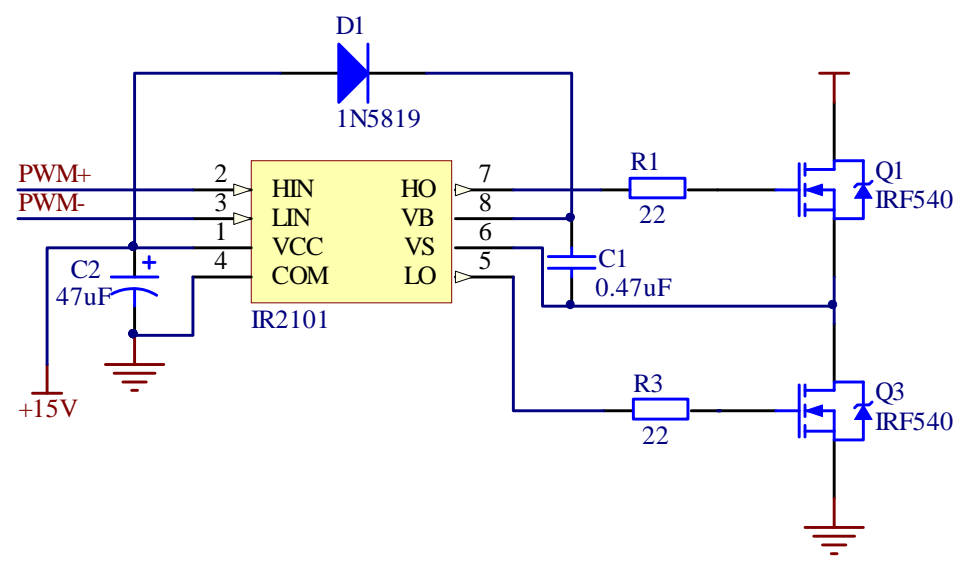

Fig.2 MOSFET driver circuit

As shown in Figure2, a piece of IR2101 drives the side-arm of the H-bridge. Two reverse phase PWM signals generated by the microcontroller are respectively connected to the two inputs of the HIN and LIN of the IR2101, and are amplified to approximately 15 volts by the MOSFET driver. The Gate of the MOSFET is driven by two output terminals HO and LO. The lower bridge leg is grounded and Q3 can be switched on and off directly from the output LO. While the upper bridge arm is suspended, Q1's Source is connected to the VS pin. A bootstrap capacitor C1 is connected between VB and VS, and the power supply charges C1 through a high switching frequency Schottky diode 1N5819. This diode is used to prevent C1 reverse discharge. When the upper arm control signal HIN sets, VB and HO are turned on inside the IR2101 (IR2101 internal structure is shown in Literature [4]). On the bridge arm Q1 Gate-Source voltage is about the voltage on the bootstrap capacitor, that is, the supply voltage $15 \mathrm{~V}$ minus the diode voltage $0.6 \mathrm{~V}, 14.4$ volts, so the Q1 meet the saturation conduction conditions. When the HIN resets, HO and VS are the internal conduction, and the potentials of the Gate and Source on Q1 is the same nearly, so Q1 is off. The other side of the H-bridge is the same principle.

SPWM generator. This design uses STC15W4K16S4 internal PWM waveform generator. STC15W4K series MCU integrates 6-channel high-precision PWM waveform generator. The PWM waveform generator has a 15-bit PWM counter for six PWM applications. Users can set the initial level of each PWM and the two rollover moments in a cycle [5], and make it very flexible to change the duty cycle of each PWM. This is conducive to achieving symmetrical complementary PWM waveform output, variable frequency function and dead zone control. 
Two reverse phase SPWM signals are generated by programming. One SPWM signal is connected to the IR2101's HIN pin and other IR2101's LIN pin. Other SPWM signal is connected oppositely.

Filter design. H-bridge inverter output voltage is AC SPWM waveform and its amplitude is approximately equal to the voltage of the external DC power supply. In fact, SPWM is used to replace the sine wave with a set of pulses that are same amplitude but different width. In addition to containing the modulation signal, SPWM signal also contains a high frequency carrier and the harmonic component near carrier double-frequency, but almost no other harmonics, in particular, no the low-order harmonic close to fundamental wave. If the parameters of $\mathrm{RC}$ low-pass filter are appropriate, the sine wave can be gotten at the LPF output terminal. According to the RC LPF cut-off frequency $\mathrm{f}=1 / 2 \pi \mathrm{RC}$, when $\mathrm{R}=100 \Omega$ and $\mathrm{C}=0.47 \mu \mathrm{F}$, the cutoff frequency is $3386 \mathrm{~Hz}$, which can meet the requirements that LPF output sine wave from $2400 \mathrm{~Hz}$ to $3000 \mathrm{~Hz}$.

\section{Program Design of AC Supply System}

SPWM signal generation and frequency conversion function. The computing capability of a microcontroller is limited so that it spends a lot of time to do mathematical operations. So the duty ratio numbers that change according to the sine law are stored in the flash memory beforehand, and it be called "sine table", as shown in Figure 3. A cycle of sine wave is divided into 40 points, in other words, a sine wave cycle contains 40 PWM wave periods.

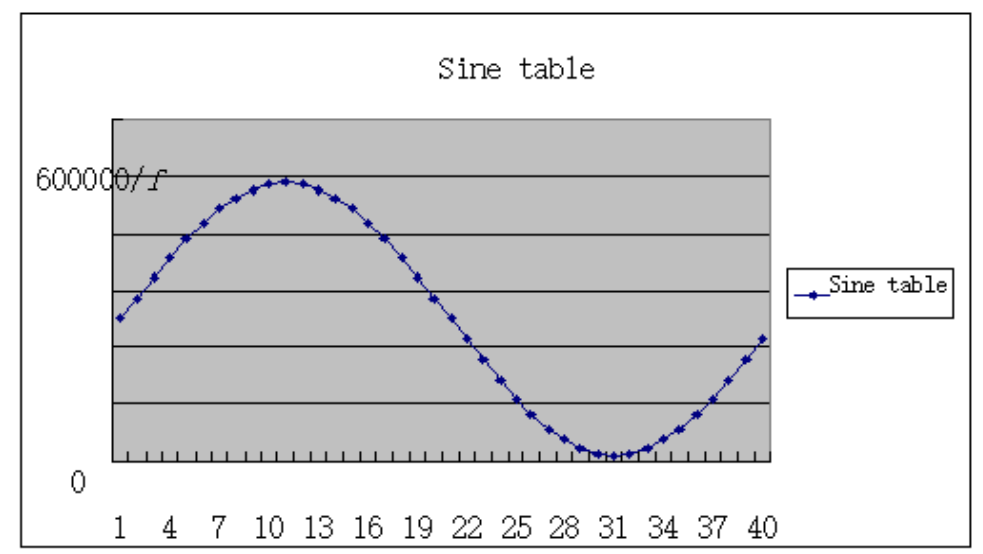

Fig.3 Sine table generated by Excel

When the PWM counter clock is selected as $24 \mathrm{MHz}$ of the system clock frequency, assuming the frequency of the sine wave is $f$, the count of the PWM counter is $N$, then the PWM wave period is $N$ clock periods, i.e., $\frac{N}{24 \times 10^{6}}$. Due to the sine wave cycle is equal to $40 \mathrm{PWM}$ wave periods; the equation can be listed as

$$
\frac{1}{f}=40 \times \frac{N}{24 \times 10^{6}} .
$$

$N$ can be solved by $f$.

Sine table generated by the Excel, the peak value is slightly less than $N$, in order to facilitate the setting of dead zone. In the Excel, the sequence numbers of 0-39 put in the column A, and the 
amplitude which is half of the peak-peak value put in the column B. The column C sets the offset. Use the formula

$$
D_{i}=B_{i} \times \sin \left(\frac{2 \pi A_{i}}{40}\right)+C_{i}, 0 \leq i \leq 39
$$

here $i$ is the number of rows, to calculate the sine table values in the column D.

From $2400 \mathrm{~Hz}$ to $3000 \mathrm{~Hz}$, take values at $50 \mathrm{~Hz}$ interval as the sine wave frequency $f$ and use the above method to generate 13 sine tables. The program achieves frequency conversion function through inquiring about different sine tables.

Dead zone setting. As shown in Figure 4, dead zone is the protection time. Because the switching speeds of the upper MOSFET and the lower MOSFET of the same side of the H-bridge is different, there is the danger of short circuit. According to the datasheet, the turn-on delay time of MOSFET IRF540N is $11 \mathrm{~ns}$, and the turn-off delay time is $39 \mathrm{~ns}$ [6], and the turn-off time is slower than the turn-on time. So the "dead zone" time has to be set to ensure that after the upper (or lower) MOSFET of the same side of the bridge is completely turned off, and then the lower (or upper) MOSFET is turned on. This prevents the short-circuiting of same side of the bridge. The dead zone time is set to 3 clocks. When the clock frequency is $24 \mathrm{MHz}$, the time is $125 \mathrm{~ns}$. This ensures that two MOSFET of same side of the bridge will not turn on at the same time.

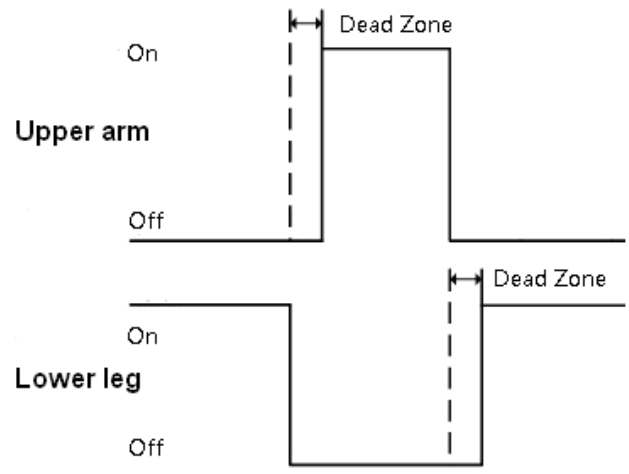

Fig.4 "Dead zone" diagram

\section{Conclusions}

The SPWM outputs are realized by two enhanced PWM controllers of the STC15W4K16S4 microcontroller, which drive the H-bridge inverter circuit to realize the conversion of DC to AC supply within 100 volts. It can output $2400 \sim 3000 \mathrm{~Hz}$ sinusoidal alternating current, and realizes the adjustable frequency at $50 \mathrm{~Hz}$ step size. So it can be used as the excitation source of metal wear particle detection sensor.

\section{Reference}

[1] YAN Hongzhi and ZHANG Yijun: Chinese Journal of Sensors and Actuators, No.4 (2002):p. 334

[2] Haiying Jiang, Ping Gao and Wenjun Zhao: Advances in Engineering Research Vol. 51 Part 1 (2016), p. 88 
[3] JIANG Lin and XIAO Wei: Chinese Journal of Electronic Design Engineering, No. 11 (2010), p. 27

[4] Ir2101 Data Sheet on http://www.infineon.com

[5] STC15series MCU Data Sheet on http:// www. stcmcu.com

[6] IRF540N Data Sheet on http://www.infineon.com 